\title{
Symmetry Methods of Flow and Heat Transfer between Slowly Expanding or Contracting Walls
}

\author{
Gabriel Magalakwe and Chaudry Masood Khalique \\ International Institute for Symmetry Analysis and Mathematical Modelling, Department of Mathematical Sciences, \\ North-West University, Mafikeng Campus, Private Bag X2046, Mmabatho 2735, South Africa
}

Correspondence should be addressed to Chaudry Masood Khalique; masood.khalique@nwu.ac.za

Received 21 April 2013; Accepted 21 May 2013

Academic Editor: H. Jafari

Copyright ( 2013 G. Magalakwe and C. M. Khalique. This is an open access article distributed under the Creative Commons Attribution License, which permits unrestricted use, distribution, and reproduction in any medium, provided the original work is properly cited.

\begin{abstract}
An analysis has been carried out for the flow and heat transfer of an incompressible laminar and viscous fluid in a rectangular domain bounded by two moving porous walls which enable the fluid to enter or exit during successive expansions or contractions. The basic equations governing the flow are reduced to the ordinary differential equations using Lie-group analysis. Effects of the permeation Reynolds number $R_{e}$, porosity $R$, and the dimensionless wall dilation rate $\alpha$ on the self-axial velocity are studied both analytically and numerically. The solutions are represented graphically. The analytical procedure is based on double perturbation in the permeation Reynolds number $R_{e}$ and the wall expansion ratio $\alpha$, whereas the numerical solution is obtained using Runge-Kutta method with shooting technique. Results are correlated and compared for some values of the physical parameters. Lastly, we look at the temperature distribution.
\end{abstract}

\section{Introduction}

The studies on the boundary layer flow and heat transfer over a stretching surface have become more and more prominent in a number of engineering applications. For instance, during extrusion of a polymer sheet, the reduction of both thickness and width takes place in a cooling bath. The quality of the final product depends upon the heat transfer rate at the stretching surface. In the past, many experimental and theoretical attempts on this topic have been made. Such studies have been presented under the various assumptions of small Reynolds number $R_{e}$, intermediate $R_{e}$, large $R_{e}$, and arbitrary $R_{e}$. The steady flow in a channel with stationary walls and small $R_{e}$ has been studied by Berman [1]. Dauenhauer and Majdalani [2] numerically discussed the two-dimensional viscous flow in a deformable channel when $-50<R_{e}<200$ and $-100<\alpha<$ 100 ( $\alpha$ denotes the wall expansion ratio). Majdalani et al. [3] analyzed the channel flow of slowly expanding-contracting walls which leads to the transport of biological fluids. They first derived the analytic solution for small $R_{e}$ and $\alpha$ and then compared it with the numerical solution.
The flow problem given in study [3] has been analytically solved by Boutros et al. [4] when $R_{e}$ and $\alpha$ vary in the ranges $-5<R_{e}<5$ and $-1<\alpha<1$. They used the Lie-group method in this study. Mahmood et al. [5] discussed the homotopy perturbation and numerical solutions for viscous flow in a deformable channel with porous medium. Asghar et al. [6] computed exact solution for the flow of viscous fluid through expanding-contracting channels. They used symmetry methods and conservation laws.

The flow and heat transfer in square domain have been studied by Noor et al. [7]. Our main aim is to study the heat transfer in a rectangular domain. In this study, symmetry methods are applied to a natural convection boundary layer problem. The main advantage of such a method is that it can successfully be applied to a nonlinear differential equation. The symmetries of differential equations are those groups of transformation under which the differential equation remains invariant, that is, a symmetry group maps any solution to any other solutions. The symmetry solutions are quite popular because they result in reductions of independent variable of the problem. 
The purpose of this paper is to generalize the flow analysis and heat distribution of [4]. The salient features have been taken into account when the fluid saturates the porous medium. Like in [4], the analytic solution for the arising nonlinear flow problem is given by employing the Lie-group method (with $R_{e}$ and $\alpha$ as the perturbation quantities). Finally, the graphs of velocity and temperature are plotted and discussed.

\section{Mathematical Formulation of the Problem}

Let us consider a rectangular domain bounded by two walls of equal permeability that enable the fluid to enter or exit during successive expansions or contractions. The walls expand or contract uniformly at the time-dependent rate $\dot{h}$. The continuous sheet aligned with the $x$-axis at $y=0$ means that the wall is impulsively stretched with the velocity $U_{w}$ along the $x$-axis and $T_{w}(x, t)$ as our surface temperature. At $y=h(t)$, it is assumed that the fluid inflow velocity $V_{w}$ is independent of the position. A thin fluid film with uniform thickness $h(t)$ rests on the horizontal wall. The governing time-dependent equations for mass, momentum, and energy are given by

$$
\begin{gathered}
\frac{\partial \bar{u}}{\partial \bar{x}}+\frac{\partial \bar{v}}{\partial \bar{y}}=0 \\
\frac{\partial \bar{u}}{\partial t}+\bar{u} \frac{\partial \bar{u}}{\partial \bar{x}}+\bar{v} \frac{\partial \bar{u}}{\partial \bar{y}}=-\frac{1}{\rho} \frac{\partial \bar{P}}{\partial \bar{x}}+v\left[\frac{\partial^{2} \bar{u}}{\partial \bar{x}^{2}}+\frac{\partial^{2} \bar{u}}{\partial \bar{y}^{2}}\right]-\frac{\nu \phi}{k} \bar{u}, \\
\frac{\partial \bar{v}}{\partial t}+\bar{u} \frac{\partial \bar{v}}{\partial \bar{x}}+\bar{v} \frac{\partial \bar{v}}{\partial \bar{y}}=-\frac{1}{\rho} \frac{\partial \bar{P}}{\partial \bar{y}}+v\left[\frac{\partial^{2} \bar{v}}{\partial \bar{x}^{2}}+\frac{\partial^{2} \bar{v}}{\partial \bar{y}^{2}}\right] \\
-\frac{v \phi}{k} \bar{v}+g \beta\left(T-T_{w}\right), \\
\frac{\partial T}{\partial t}+\bar{u} \frac{\partial T}{\partial \bar{x}}+\bar{v} \frac{\partial T}{\partial \bar{y}}=\alpha\left[\frac{\partial^{2} T}{\partial \bar{x}^{2}}+\frac{\partial^{2} T}{\partial \bar{y}^{2}}\right],
\end{gathered}
$$

where $\bar{u}$ and $\bar{v}$ are the velocity components in the $\bar{x}$ and $\bar{y}$ directions, respectively, and $T$ is temperature. We assume that the fluid properties are constant. Here, $\rho$ is the fluid density, $\mu$ is the dynamic viscosity, and $\bar{k}$ is the thermal conductivity of an incompressible fluid. Thus, the kinematic viscosity is $\nu=\mu / \rho, g$ is the acceleration due to gravity, $\beta$ is the coefficient of the thermal expansion, and the thermal diffusivity is $\alpha=$ $\bar{k} / \rho c_{p}$, where $c_{p}$ is the specific heat, $\bar{P}$ is the pressure, $t$ is time, and $\phi$ and $k$ are the porosity and permeability of porous medium, respectively.

The appropriate boundary conditions are

$$
\begin{aligned}
& \text { (i) } \bar{u}=0, \quad \bar{v}=-V_{w}, \quad T=T_{w} \quad \text { at } \bar{y}=h(t), \\
& \text { (ii) } \frac{\partial \bar{u}}{\partial \bar{y}}=0, \quad \bar{v}=0, \quad \frac{\partial T}{\partial \bar{y}}=0 \quad \text { at } \bar{y}=0, \\
& \text { (iii) } \bar{u}=0 \quad \text { at } \bar{x}=0,
\end{aligned}
$$

where $h(t)$ is the film thickness. The boundary condition reflects that the fluid motion within the liquid film is caused by the viscous shear arising from the stretching of the elastic wall.

Now we will express the axial velocity, normal velocity, and boundary conditions in terms of the stream function $\bar{\Psi}$. From the continuity Equation (1), there exists a dimensional stream function $\bar{\Psi}(\bar{x}, \bar{y}, t)$ such that

$$
\bar{u}=\frac{\partial \bar{\Psi}}{\partial \bar{y}}, \quad \bar{v}=-\frac{\partial \bar{\Psi}}{\partial \bar{x}},
$$

which satisfies (1) identically.

Introducing the dimensionless normal coordinate $y=$ $\bar{y} / h(t),(6)$ becomes

$$
\bar{u}=\frac{1}{h} \frac{\partial \bar{\Psi}}{\partial y}, \quad \bar{v}=-\frac{\partial \bar{\Psi}}{\partial \bar{x}} .
$$

Substituting (7) into (2)-(4), we obtain

$$
\begin{gathered}
h^{2} \bar{\Psi}_{y t}-h \dot{h} y \bar{\Psi}_{y y}-h \dot{h} \bar{\Psi}_{y}+h \bar{\Psi}_{y} \bar{\Psi}_{\bar{x} y}-h \bar{\Psi}_{\bar{x}} \bar{\Psi}_{y y} \\
=-\frac{h^{3}}{\rho} \bar{P}_{\bar{x}}+\nu\left[h^{2} \bar{\Psi}_{\bar{x} \bar{x} y}+\bar{\Psi}_{y y y}\right]-\frac{h^{2} \nu \phi}{k} \bar{\Psi}_{y}, \\
-h^{2} \bar{\Psi}_{\bar{x} t}+h \dot{h} y \bar{\Psi}_{\bar{x} y}-h \bar{\Psi}_{y} \bar{\Psi}_{\bar{x} \bar{x}}+h \bar{\Psi}_{\bar{x}} \bar{\Psi}_{\bar{x} y} \\
=-\frac{h}{\rho} \bar{P}_{y}+\nu\left[-h^{2} \bar{\Psi}_{\bar{x} \bar{x} \bar{x}}-\bar{\Psi}_{\bar{x} y y}\right] \\
+\frac{h^{2} \nu \phi}{k} \bar{\Psi}_{x}+g \beta\left(T-T_{w}\right) h^{2}, \\
\frac{\partial T}{\partial t}+\frac{1}{h} \bar{\Psi}_{y} \frac{\partial T}{\partial \bar{x}}-\bar{\Psi}_{\bar{x}} \frac{\partial T}{\partial \bar{y}}=\alpha\left[\frac{\partial^{2} T}{\partial \bar{x}^{2}}+\frac{\partial^{2} T}{\partial \bar{y}^{2}}\right],
\end{gathered}
$$

where a dot denotes the derivative with respect to $t$.

The variables in (8) are dimensionless according to

$$
\begin{gathered}
u=\frac{\bar{u}}{V_{w}}, \quad v=\frac{\bar{u}}{V_{w}}, \quad x=\frac{\bar{x}}{h(t)}, \\
y=\frac{\bar{y}}{h(t)}, \quad \Psi=\frac{\bar{\Psi}}{h V_{w}}, \quad P=\frac{\bar{P}}{\rho V_{w}^{2}}, \\
t=\frac{\bar{t}}{h V_{w}}, \quad \alpha=\frac{h \dot{h}}{v}, \quad \theta=\frac{T-T_{h}}{T_{w}-T_{h}}, \quad \frac{1}{R}=\frac{v \phi a}{k V_{w}} .
\end{gathered}
$$

Substituting (9) into (8), we have

$$
\begin{aligned}
\Psi_{y \bar{t}} & \Psi_{y} \Psi_{x y}-\Psi_{x} \Psi_{y y}+P_{x} \\
& -\frac{1}{R_{e}}\left[\alpha \Psi_{y}+\alpha y \Psi_{y y}+\Psi_{x x y}+\Psi_{y y y}\right]-\frac{1}{R} \Psi_{y}=0 \\
\Psi_{x \bar{t}} & \Psi_{y} \Psi_{x x}-\Psi_{x} \Psi_{x y}-P_{y} \\
& -\frac{1}{R_{e}}\left[\alpha y \Psi_{x y}+\Psi_{x y y}+\Psi_{x x x}\right]+\frac{1}{R} \Psi_{x}+\frac{1}{h^{2}} G_{r} \theta=0 \\
\frac{\partial \theta}{\partial \bar{t}}+ & \Psi_{y} \frac{\partial \theta}{\partial x}-\Psi_{x} \frac{\partial \theta}{\partial y}=\frac{1}{P_{r} R_{e}}\left[\frac{\partial^{2} \theta}{\partial x^{2}}+\frac{\partial^{2} \theta}{\partial y^{2}}\right]
\end{aligned}
$$

in which $R_{e}=h V_{w} / v$ is the permeation Reynolds number, 
$G_{r}=g \beta\left(T_{w}-T_{h}\right) h^{3} / v^{2}$ is the Grashof number, $P_{r}=v / \alpha$ is the Prandtl number, and $\dot{h}=\alpha v / h$.

Through (7) and (9), we have

$$
u=\frac{\partial \Psi}{\partial y}, \quad v=-\frac{\partial \Psi}{\partial x},
$$

and, thus, the boundary conditions take the following forms:

$$
\begin{gathered}
\Psi_{y}=0, \quad \Psi_{x}=1, \quad \theta=1 \quad \text { at } y=1, \\
\Psi_{y y}=0, \quad \Psi_{x}=0, \quad \theta_{y}=0 \quad \text { at } y=0, \\
\Psi_{y}=0 \quad \text { at } x=0 .
\end{gathered}
$$

\section{Solution of the Problem}

This section derives the similarity solutions using Lie-group method under which (10)-(12) are invariant.

3.1. Lie Symmetry Analysis. We consider the one-parameter

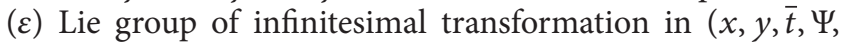
$P, \theta$ ) given by

$$
\begin{aligned}
x^{*} & =x+\varepsilon \phi(x, y, \bar{t}, \Psi, P, \theta)+0\left(\varepsilon^{2}\right), \\
y^{*} & =y+\varepsilon \zeta(x, y, \bar{t}, \Psi, P, \theta)+0\left(\varepsilon^{2}\right), \\
t^{*} & =\bar{t}+\varepsilon F(x, y, \bar{t}, \Psi, P, \theta)+0\left(\varepsilon^{2}\right), \\
\Psi^{*} & =\Psi+\varepsilon \eta(x, y, \bar{t}, \Psi, P, \theta)+0\left(\varepsilon^{2}\right), \\
P^{*} & =P+\varepsilon g(x, y, \bar{t}, \Psi, P, \theta)+0\left(\varepsilon^{2}\right), \\
\theta^{*} & =\theta+\varepsilon H(x, y, \bar{t}, \Psi, P, \theta)+0\left(\varepsilon^{2}\right),
\end{aligned}
$$

with $\varepsilon$ as a small parameter.

In view of Lie's algorithm, the vector field is

$$
X=\phi \frac{\partial}{\partial x}+\zeta \frac{\partial}{\partial y}+F \frac{\partial}{\partial \vec{t}}+\eta \frac{\partial}{\partial \Psi}+g \frac{\partial}{\partial P}+H \frac{\partial}{\partial \theta},
$$

if it is left variant by the transformation $(x, y, \bar{t}, \Psi, P, \theta) \rightarrow$ $\left(x^{*}, y^{*}, t^{*}, \Psi^{*}, P^{*}, \theta^{*}\right)$.

The solutions $\Psi=\Psi(x, y, \bar{t}), P=P(x, y, \bar{t})$ and $\theta=\theta(x$, $y, \bar{t})$ are invariant under the symmetry (16) if

$$
\begin{aligned}
& \Phi_{\Psi}=X(\Psi-\Psi(x, y, \bar{t}))=0, \quad \text { where } \Psi=\Psi(x, y, \bar{t}), \\
& \Phi_{P}=X(P-P(x, y, \bar{t}))=0, \quad \text { where } P=P(x, y, \bar{t}), \\
& \Phi_{\theta}=X(\theta-\theta(x, y, \bar{t}))=0, \quad \text { where } \theta=\theta(x, y, \bar{t}) .
\end{aligned}
$$

We set

$$
\begin{aligned}
\Delta_{1}= & \Psi_{y \bar{t}}+\Psi_{y} \Psi_{x y}-\Psi_{x} \Psi_{y y}+P_{x} \\
& -\frac{1}{R_{e}}\left[\alpha \Psi_{y}+\alpha y \Psi_{y y}+\Psi_{x x y}+\Psi_{y y y}\right]-\frac{1}{R} \Psi_{y}, \\
\Delta_{2}= & \Psi_{x \bar{t}}+\Psi_{y} \Psi_{x x}-\Psi_{x} \Psi_{x y}-P_{y} \\
& -\frac{1}{R_{e}}\left[\alpha y \Psi_{x y}+\Psi_{x y y}+\Psi_{x x x}\right]+\frac{1}{R} \Psi_{x}+\frac{1}{h^{2}} G_{r} \theta, \\
\Delta_{3}=\frac{\partial \theta}{\partial \bar{t}}+ & \Psi_{y} \frac{\partial \theta}{\partial x}-\Psi_{x} \frac{\partial \theta}{\partial y}-\frac{1}{P_{r} R_{e}}\left[\frac{\partial^{2} \theta}{\partial x^{2}}+\frac{\partial^{2} \theta}{\partial y^{2}}\right] .
\end{aligned}
$$

The vector field $X$ given by (16) is a symmetry generator of (10)-(12) if and only if

$$
\left.X^{[3]}\left(\Delta_{j}\right)\right|_{\Delta_{j}=0}=0, \quad j=1,2,3,
$$

in which

$$
\begin{aligned}
X^{[3]}= & \phi \frac{\partial}{\partial x}+\zeta \frac{\partial}{\partial y}+F \frac{\partial}{\partial \bar{t}}+\eta \frac{\partial}{\partial \Psi}+g \frac{\partial}{\partial P}+H \frac{\partial}{\partial \theta} \\
& +\eta^{x} \frac{\partial}{\partial \Psi_{x}}+\eta^{y} \frac{\partial}{\partial \Psi_{y}}+g^{x} \frac{\partial}{\partial P_{x}}+g^{y} \frac{\partial}{\partial P_{y}} \\
& +H^{x} \frac{\partial}{\partial \theta_{x}}+H^{y} \frac{\partial}{\partial \theta_{y}}+H^{\bar{t}} \frac{\partial}{\partial \theta_{\bar{t}}}+\eta^{x y} \frac{\partial}{\partial \Psi_{x y}} \\
& +\eta^{x \bar{t}} \frac{\partial}{\partial \Psi_{x \bar{t}}}+\eta^{y \bar{t}} \frac{\partial}{\partial \Psi_{y \bar{t}}}+\eta^{x x} \frac{\partial}{\partial \Psi_{x x}}+\eta^{y y} \frac{\partial}{\partial \Psi_{y y}} \\
& +H^{x x} \frac{\partial}{\partial \theta_{x x}}+H^{y y} \frac{\partial}{\partial \theta_{y y}}+\eta^{x x y} \frac{\partial}{\partial \Psi_{x x y}} \\
& +\eta^{x y y} \frac{\partial}{\partial \Psi_{x y y}}+\eta^{x x x} \frac{\partial}{\partial \Psi_{x x x}}+\eta^{y y y} \frac{\partial}{\partial \Psi_{y y y}}
\end{aligned}
$$

is the third prolongation of $X$.

We now introduce the total derivatives by differentiating (15) with respect to $x, y$, and $\bar{t}$ and construct

$$
\begin{aligned}
D_{x}= & \partial_{x}+\Psi_{x} \partial_{\Psi}+P_{x} \partial_{p}+\theta_{x} \partial_{\theta}+\Psi_{x x} \partial_{\Psi_{x}} \\
& +P_{x x} \partial_{P_{x}}+\theta_{x x} \partial_{\theta_{x}}+\Psi_{x y} \partial_{\Psi_{y}}+\theta_{x y} \partial_{\theta_{y}}+\cdots, \\
D_{y}= & \partial_{y}+\Psi_{y} \partial_{\Psi}+P_{y} \partial_{P}+\theta_{y} \partial_{\theta}+\Psi_{y y} \partial_{\Psi_{y}} \\
& +P_{y y} \partial_{P_{y}}+\theta_{y y} \partial_{\theta_{y}}+\Psi_{x y} \partial_{\Psi_{x}}+\theta_{x y} \partial_{\theta_{x}}+\cdots, \\
D_{\bar{t}}= & \partial_{\bar{t}}+\Psi_{\bar{t}} \partial_{\Psi}+P_{\bar{t}} \partial_{P}+\theta_{\bar{t}} \partial_{\theta}+\Psi_{\overline{t t}} \partial_{\Psi_{\bar{t}}} \\
& +P_{\overline{t t}} \partial_{P_{\bar{t}}}+\theta_{\overline{t t}} \partial_{\theta_{\bar{t}}}+\Psi_{x \bar{t}} \partial_{\Psi_{x}}+\theta_{x \bar{t}} \partial_{\theta_{x}}+\cdots .
\end{aligned}
$$


Choosing small $G_{r}$ when $T_{h} \approx T_{w}$, the system of (10)-(12) has the six parameter Lie-group points of symmetries generated by

$$
\begin{gathered}
X_{1}=\frac{\partial}{\partial \bar{t}}, \quad X_{2}=\theta \frac{\partial}{\partial \bar{t}}, \quad X_{3}=\frac{\partial}{\partial \theta}, \quad X_{4}=\frac{\partial}{\partial \Psi}, \\
X_{5}=F_{2}(\bar{t}) \frac{\partial}{\partial y}, \quad X_{6}=F_{1}(\bar{t}) \frac{\partial}{\partial x} .
\end{gathered}
$$

3.2. Invariant Solution. When calculating invariant solutions under the group generators $X_{3}$ and $X_{4}$, we found that there are no invariant solutions. Then $X_{5}$ and $X_{6}$ give solutions of (1)-(3) and this contradicts the boundary conditions.

For $X_{1}$ and $X_{2}$, the characteristic $\Phi=\left(\Phi_{\Psi}, \Phi_{P}, \Phi_{\theta}\right)$ has the following components:

$$
\Phi_{\Psi}=-\Psi_{\bar{t}}, \quad \Phi_{P}=-P_{\bar{t}}, \quad \Phi_{\theta}=-\theta_{\bar{t}} .
$$

The general solutions of invariant surface conditions (17) are given by

$$
\Psi=h(y) H(x, y), \quad P=\Gamma(x, y), \quad \theta=\tau(x, y) .
$$

Invoking (24) into (10), we have

$$
\begin{aligned}
& -K \frac{d^{3} h}{d y^{3}}+\left[-\alpha K y-h K_{1}-3 K K_{2}\right] \frac{d^{2} h}{d y^{2}} \\
& +\left[-\alpha K-2 \alpha K y K_{2}-h K_{3}\right. \\
& \left.+h K_{4}-K K_{5}-3 K K_{6}+\frac{1}{R}\right] \frac{d h}{d y}, \\
& K_{1}\left(\frac{d h}{d y}\right)^{2}+\left[-\alpha K K_{2}+\frac{1}{R} K_{2}-\alpha K K_{6} y-K K_{9}-K K_{10}\right] h \\
& +\left[K_{7}-K_{8}\right] h^{2}+\frac{1}{H} \frac{d \Gamma}{d x}, \\
& K_{1}=H_{x}, \quad K_{2}=\frac{H_{y}}{H}, \quad K_{3}=\frac{H_{x} H_{y}}{H}, \\
& K_{4}=H_{x y}, \quad K_{5}=\frac{H_{x x}}{H}, \quad K_{6}=\frac{H_{y y}}{H}, \\
& K_{7}=\frac{H_{y} H_{x y}}{H}, \quad K_{8}=\frac{H_{x} H_{y y}}{H}, \quad K_{9}=\frac{H_{x x y}}{H}, \\
& K_{10}=\frac{H_{y y y}}{H}, \quad K=R_{e} .
\end{aligned}
$$

Integration of $H_{x}=K_{1}$ from (26) leads to the following expression:

$$
H(x, y)=x K_{1}(y)+K_{11}(y) \text {. }
$$

The above equation when used into $\Psi=h(y) H(x, y)$ (from (24)) gives

$$
\Psi=\left(x K_{1}(y)+K_{11}(y)\right) h(y),
$$

which after differentiating with respect to $y$ and using (14c) yields

$$
K_{11}(y) h(y)=K_{12},
$$

where $K_{12}$ is a constant of integration and hence (28) reads

$$
\Psi=x G(y)+K_{12}
$$

with $G(y)=K_{1}(y) h(y)$.

Putting $P=\Gamma(x, y)$ from (24) and (27) into the last term of (25) yields

$$
K_{11}=0 .
$$

With the help of (27) and (31), one obtains

$$
H(x, y)=x K_{1}(y),
$$

while (29)-(31) yield

$$
\Psi=x G(y)
$$

Due to (13) and (33), one may write

$$
u=x \frac{d G}{d y}, \quad v=-G .
$$

Using (33) in (11) and then differentiating with respect to $x$, one arrives at the following result:

$$
P_{x y}=\frac{1}{h^{2}} G_{r} \theta_{x} .
$$

Putting (33) into (10), differentiating with respect to $y$, and then using (35), we obtain

$$
\begin{aligned}
\frac{d^{4} G}{d y^{4}} x & +\alpha\left[y \frac{d^{3} G}{d y^{3}}+2 \frac{d^{2} G}{d y^{2}}\right] x+R_{e} G \frac{d^{3} G}{d y^{3}} x \\
- & R_{e} \frac{d G}{d y} \frac{d^{2} G}{d y^{2}} x-R_{e} \frac{d^{2} G}{d y^{2}} \frac{1}{R} x+\frac{1}{h^{2}} G_{r} \theta_{x}=0 .
\end{aligned}
$$

Using (33) and $\theta=\tau(x, y)$ from (24) in (12), we can write

$$
x \frac{d G}{d y} \frac{\partial \tau}{\partial x}-G \frac{\partial \tau}{\partial y}-\frac{1}{P_{r} R_{e}}\left[\frac{\partial^{2} \tau}{\partial x^{2}}+\frac{\partial^{2} \tau}{\partial y^{2}}\right]=0,
$$

and the boundary conditions (14a), (14b), and (14c) become
(i) $\frac{d G(1)}{d y}=0$,
(ii) $G(1)=1$,
(iii) $\frac{d^{2} G(0)}{d y^{2}}=0$,
(iv) $G(0)=0$,
(v) $\tau(x, 1)=0$
(vi) $\tau(x, 0)=1$.

Using $\theta=\tau(x, y)$ and equating-like powers of $h$, (36) helps in writing the following equations:

$$
\begin{gathered}
\frac{d^{4} G}{d y^{4}}+\alpha\left[y \frac{d^{3} G}{d y^{3}}+2 \frac{d^{2} G}{d y^{2}}\right]+R_{e} G \frac{d^{3} G}{d y^{3}}-R_{e} \frac{d G}{d y} \frac{d^{2} G}{d y^{2}} \\
-R_{e} \frac{d^{2} G}{d y^{2}} \frac{1}{R}=0, \\
G_{r} \tau_{x}=0 .
\end{gathered}
$$


The above equation implies that $\tau=E(y)$ and $G_{r}=0$ which satisfy our assumption that $G_{r}$ is very small. Now (36)-(38) yield

$$
\begin{gathered}
\frac{d^{4} G}{d y^{4}}+\alpha\left[y \frac{d^{3} G}{d y^{3}}+2 \frac{d^{2} G}{d y^{2}}\right]+R_{e} G \frac{d^{3} G}{d y^{3}} \\
-R_{e} \frac{d G}{d y} \frac{d^{2} G}{d y^{2}}-R_{e} \frac{d^{2} G}{d y^{2}} \frac{1}{R}=0, \\
G(y) \frac{\partial E}{\partial y}+\frac{1}{P_{r} R_{e}}\left[\frac{\partial^{2} E}{\partial y^{2}}\right]=0, \\
\begin{array}{ll}
\text { (i) } \frac{d G(1)}{d y}=0, \quad \text { (ii) } G(1)=1, \quad \text { (iii) } \frac{d^{2} G(0)}{d y^{2}}=0, \\
\text { (iv) } G(0)=0, \quad \text { (v) } E(1)=1, \quad \text { (vi) } E^{\prime}(0)=0 .
\end{array}
\end{gathered}
$$

3.3. Analytical Solution. The aim of this section is to find the solutions of (40)-(42) using double perturbation $[3,4]$. For small $R_{e}$ and $\alpha$, we expand

$$
\begin{gathered}
G=G_{1}+R_{e} G_{2}+O\left(R_{e}^{2}\right), \\
G_{1}=G_{10}+\alpha G_{11}+O\left(\alpha^{2}\right), \\
G_{2}=G_{20}+\alpha G_{21}+O\left(\alpha^{2}\right) .
\end{gathered}
$$

Using (43) into (40)-(42) and then solving the resulting problems for small $R_{e}$ and $\alpha$, we obtain

$$
\begin{gathered}
G_{10}(y)=-\frac{1}{2} y^{3}+\frac{3}{2} y, \\
G_{11}(y)=\frac{3}{40} y^{5}-\frac{3}{20} y^{3}+\frac{3}{40} y, \\
G_{20}(y)=\frac{1}{280} y^{7}-\frac{3}{280} y^{3}+\frac{1}{140} y \\
+\frac{1}{R}\left(-\frac{1}{40} y^{5}+\frac{1}{20} y^{3}-\frac{1}{40} y\right), \\
G_{21}(y)=-\frac{13}{20160} y^{9}-\frac{9}{2800} y^{7}+\frac{9}{5600} y^{5} \\
+\frac{227}{25200} y^{3}+\frac{227}{33600} y \\
+\frac{1}{R}\left(\frac{1}{210} y^{7}-\frac{3}{200} y^{5}+\frac{11}{700} y^{3}-\frac{23}{4200} y\right), \\
G_{1}(y)=-\frac{1}{2} y^{3}+\frac{3}{2} y+\alpha\left[\frac{3}{40} y^{5}-\frac{3}{20} y^{3}+\frac{3}{40} y\right], \\
G_{2}(y)=\frac{1}{280} y^{7}-\frac{3}{280} y^{3}+\frac{1}{140} y \\
\left(-\frac{1}{40} y^{5}+\frac{1}{20} y^{3}-\frac{1}{40} y\right)
\end{gathered}
$$

$$
\begin{aligned}
+\alpha[ & -\frac{13}{20160} y^{9}-\frac{9}{2800} y^{7}+\frac{9}{5600} y^{5} \\
& +\frac{227}{25200} y^{3}-\frac{227}{33600} y \\
& \left.+\frac{1}{R}\left(\frac{1}{210} y^{7}-\frac{3}{200} y^{5}+\frac{11}{700} y^{3}-\frac{23}{4200} y\right)\right],
\end{aligned}
$$

$$
\begin{aligned}
G(y)=\left(-\frac{1}{2} y^{3}+\frac{3}{2} y+\alpha\left[\frac{3}{40} y^{5}-\frac{3}{20} y^{3}+\frac{3}{40} y\right]\right) \\
+R_{e}\left(\frac{1}{280} y^{7}-\frac{3}{280} y^{3}+\frac{1}{140} y\right. \\
+\frac{1}{R}\left(-\frac{1}{40} y^{5}+\frac{1}{20} y^{3}-\frac{1}{40} y\right) \\
+\alpha\left[-\frac{13}{20160} y^{9}-\frac{9}{2800} y^{7}+\frac{9}{5600} y^{5}\right. \\
+\frac{227}{25200} y^{3}-\frac{227}{33600} y \\
+\frac{1}{R}\left(\frac{1}{210} y^{7}-\frac{3}{200} y^{5}\right. \\
\left.\left.\left.+\frac{11}{700} y^{3}-\frac{23}{4200} y\right)\right]\right)
\end{aligned}
$$

It is noted that for $R \rightarrow \infty$, the expression of $G(y)$ in [4] is recovered.

Let

$$
E=E_{1}+R_{e} E_{2}+O\left(R_{e}^{2}\right) .
$$

From (41), (45), and (46), we obtain

$$
\begin{gathered}
\frac{d^{2} E_{1}}{d y^{2}}=0, \quad E_{1}(1)=1, \quad E_{1}^{\prime}(0)=0, \\
P_{r} G(y) \frac{d E_{1}(y)}{d y}+\frac{d^{2} E_{2}(y)}{d y^{2}}, \quad E_{2}(1)=0, \quad E_{2}^{\prime}(0)=0 .
\end{gathered}
$$

Solving the above problems and using (46), one obtains

$$
E(y)=1 \text {. }
$$

3.4. Numerical Solution. Now the numerical solution of (40)-(42) has been obtained using shooting method with Runge-Kutta scheme.

\section{Results and Discussion}

Figures 1, 2, 3, and 4 illustrate the behaviour of self-axial velocity over a range of $R$ with $R_{e}$ and $\alpha$ fixed.

Figures 1 and 2 illustrate the behaviour of self-axial velocity $u / x$ for permeation Reynolds number $R_{e}=1$ (injection) and $\alpha=0.5,-0.5$ (expansion and contraction, resp.) over a range of porosity parameter $R$. For $R>0$, these figures show 


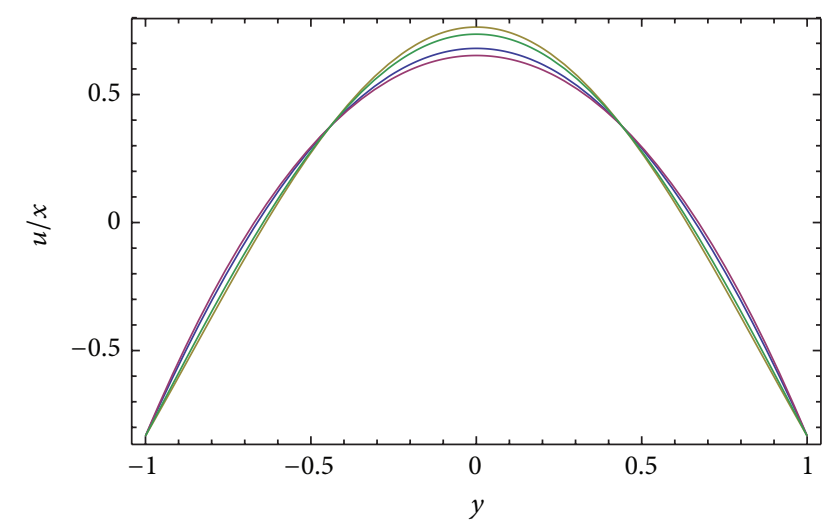

FIGURE 1: Self-axial velocity profiles over a range of $R$, where blue $=$ 1 , pink $=0.5$, yellow $=-0.5$, and green $=-1$ at $R_{e}=1$ and $\alpha=0.5$.

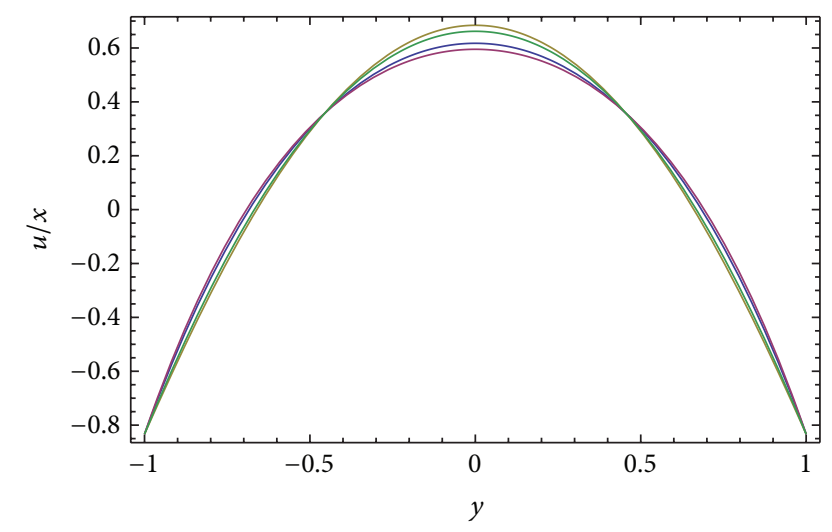

FIGURE 2: Self-axial velocity profiles over a range of $R$, where blue $=$ 1 , pink $=0.5$, yellow $=-0.5$, and green $=-1$ at $R_{e}=1$ and $\alpha=-0.5$.

that the higher porosity $R$ leads to higher self-axial velocity near the center and lower near the wall. The results for $R<0$ are quite opposite to that of $R>0$. A comparative study of these figures further indicates that the self-axial velocity near the center in case of injection with expanding wall and high porosity is higher than injection with contracting wall and high porosity.

The plots of self-axial velocity $u / x$ for permeation Reynolds number $R_{e}=-1$ (suction) and $\alpha=0.5,-0.5$ (expansion and contraction, resp.) over a range of $R$ have been displayed in Figures 3 and 4 . In case of $R>0$, these graphs depict that the higher porosity $R$ leads to lower selfaxial velocity near the center and higher near the wall. For $R<0$, these figures depict that the lower porosity $R$ leads to higher self-axial velocity near the center and lower near the wall. By comparing Figures 3 and 4, we note that the self-axial velocity near the center in case of suction with expanding wall and high porosity is higher than suction with contracting wall and high porosity.

The behaviour of the self-axial velocity $u / x$ for wall dilation rate $\alpha=-0.5$ (contraction) and $R_{e}=1,-1$ (injection and suction) over a range of $R$ has been displayed in Figures 2 and 3 . For $R>0$, Figure 3 shows that the higher the porosity $R$, the lower the self-axial velocity at the center and higher near the wall. Figure 2 shows that the higher the porosity $R$,

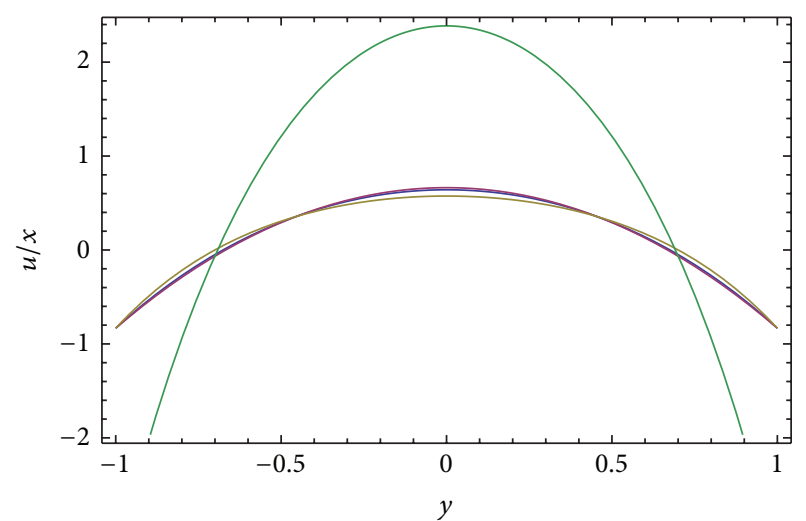

FIGURE 3: Self-axial velocity profiles over a range of $R$ where blue $=$ 1 , pink $=0.5$, yellow $=-0.5$ and green $=-1$ at $R_{e}=-1$ and $\alpha=$ -0.5 .

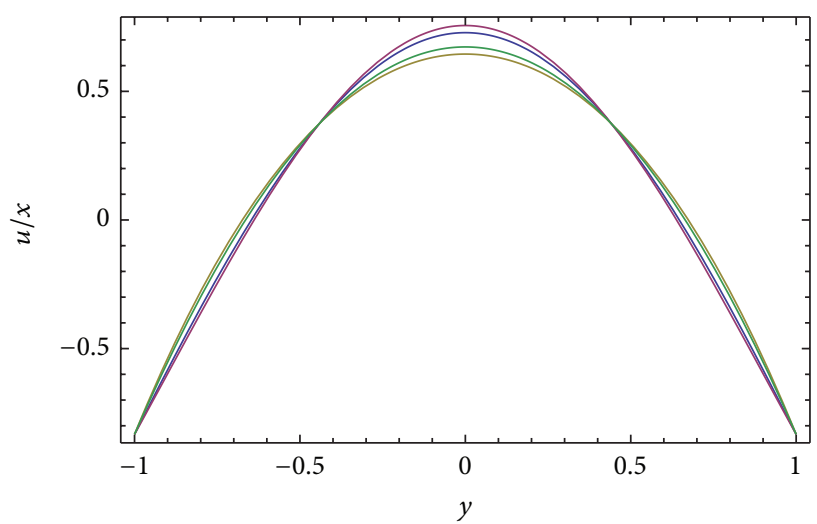

FIGURE 4: Self-axial velocity profiles over a range of $R$, where blue $=$ 1 , pink $=0.5$, yellow $=-0.5$, and green $=-1$ at $R_{e}=-1$ and $\alpha=0.5$.

the higher the self-axial velocity at the center and lower near the wall. When $R<0$, Figure 3 elucidates that the lower porosity $R$ gives a higher self-axial velocity near the center and a lower one near the wall. Figure 2 elucidates that the lower porosity $R$ gives a lower self-axial velocity near the center and a higher one near the wall. A comparative study of Figures 2 and 3 indicates that the self-axial velocity near the center in case of injection with contracting wall and high porosity is higher than suction with contracting wall and high porosity.

The variations of self-axial velocity $u / x$ for wall dilation rate $\alpha=0.5$ (expansion) and $R_{e}=1,-1$ (injection and suction) over a range of $R$ have been plotted in Figures 1 and 4 . When $R>0$, then Figure 1 shows that the higher the porosity $R$, the higher the self-axial velocity at the center and lower near the wall. Figure 4 shows that the higher the porosity $R$, the lower the self-axial velocity at the center and higher near the wall. When $R<0$, Figure 1 describes that the lower porosity $R$ gives lower self-axial velocity near the center and higher near the wall. Figure 4 provides that the lower porosity $R$ yields higher self-axial velocity near the center and lower near the wall. Comparison of Figures 1 and 4 leads to the conclusion that the self-axial velocity near the center for suction with expanding wall and high porosity is higher than injection with expanding wall and high porosity. 


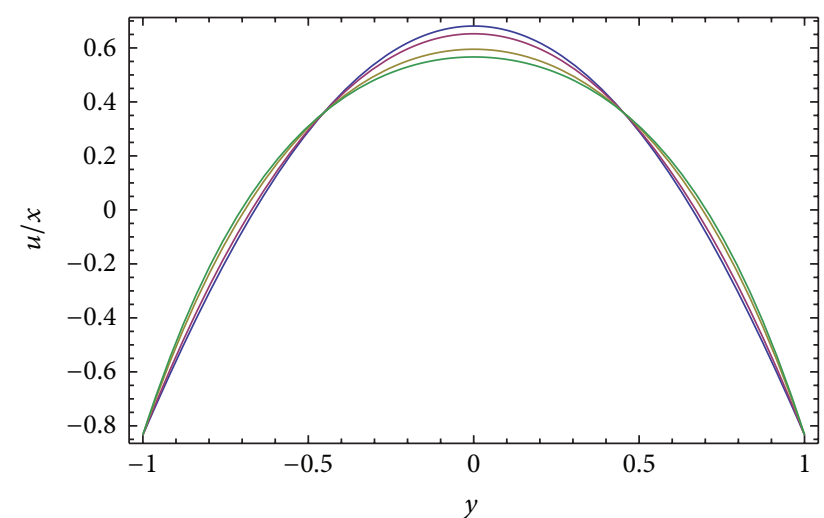

FIgURE 5: Self-axial velocity profiles over a range of $\alpha$, where blue $=$ 1 , pink $=0.5$, yellow $=-0.5$, and green $=-1$ at $R_{e}=1$ and $R=0.5$.

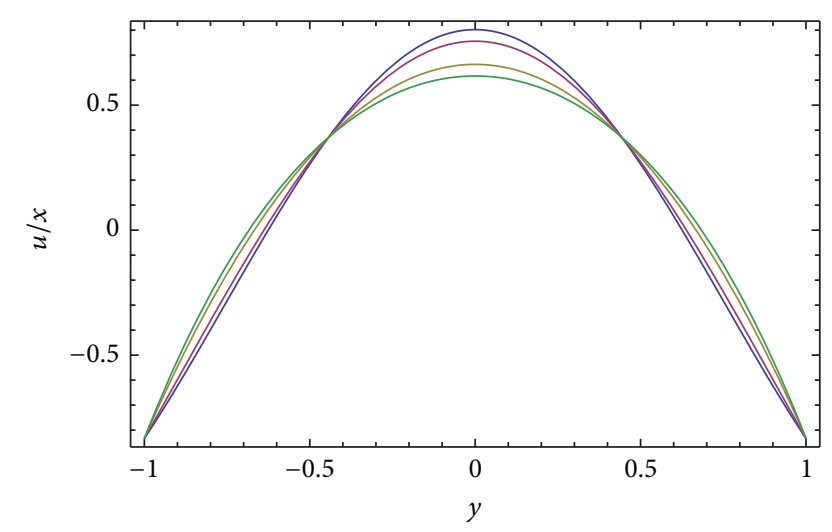

FIGURE 6: Self-axial velocity profiles over a range of $\alpha$, where blue $=$ 1 , pink $=0.5$, yellow $=-0.5$, and green $=-1$ at $R_{e}=-1$ and $R=0.5$.

Tables 1, 2, 3, and 4 depict that the percentage error decreases when $R$ increases.

Figures $5,6,7$, and 8 plot the behaviour of self-axial velocity over a range of $\alpha$ with fixed $R_{e}$ and $R$.

For $\alpha>0$, Figures 5-8 witness that the greater $\alpha$ leads to higher self-axial velocity at the center and lower near the wall. For $\alpha<0$, these figures show that an increase in contraction ratio leads to lower self-axial velocity near the center and higher near the wall. By comparing Figures 5 and 6 , we note that the self-axial velocity near the center in case of suction with expanding wall and high porosity is higher than injection with expanding wall and high porosity.

Comparison of Figures 5 and 8 shows that the self-axial velocity near the center in case of injection with expanding wall and low porosity is higher than injection with expanding wall and high porosity. Comparative study of Figures 6 and 7 reveals that the self-axial velocity near the center in case of suction with expanding wall and high porosity is higher than suction with expanding wall and low porosity. By comparing Figures 7 and 8, the self-axial velocity near the center in case of injection with expanding wall and low porosity is higher than suction with expanding wall and low porosity.

Tables 5, 6, 7, and 8 indicate that the percentage error is an increasing function of $\alpha$.

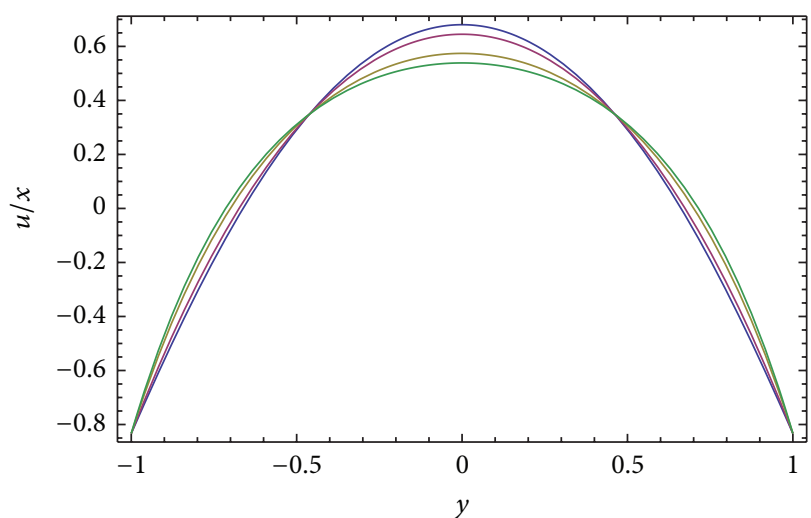

FIGURE 7: Self-axial velocity profiles over a range of $\alpha$, where blue $=$ 1 , pink $=0.5$, yellow $=-0.5$, and green $=-1$ at $R_{e}=-1$ and $R=$ -0.5 .

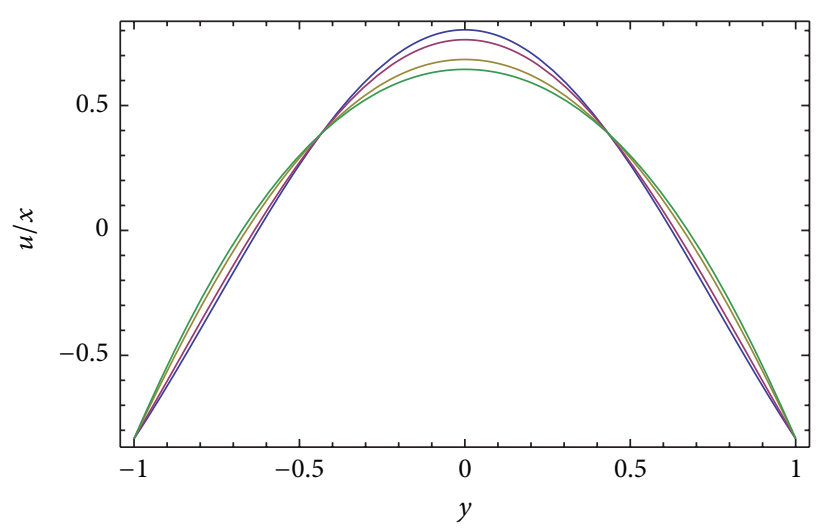

FIGURE 8: Self-axial velocity profiles over a range of $\alpha$, where blue $=$ 1 , pink $=0.5$, yellow $=-0.5$, and green $=-1$ at $R_{e}=1$ and $R=-0.5$.

Figures 9, 10, 11, and 12 illustrate the behaviour of self-axial velocity over a range of $R_{e}$ with fixed $\alpha$ and $R$.

The self-axial velocity $u / x$ for porosity parameter $R=0.5$ (high porosity) and wall dilation rate $\alpha=0.5,-0.5$ (expansion and contraction, resp.) over a range of $R_{e}$ has been sketched in Figures 9 and 10. For $R_{e}>0$, we found that increasing injection $R_{e}$ leads to a lower self-axial velocity at the center and a higher one near the wall. When $R_{e}<0$, Figures 9 and 10 indicate that increasing suction ratio leads to a higher self-axial velocity near the center and a lower one near the wall. Comparison of Figures 9 and 10 shows that the self-axial velocity near the center in case of injection with expanding wall and high porosity is higher than injection with contracting wall and high porosity.

Figures 11 and 12 provide the variation of self-axial velocity $u / x$ for porosity parameter $R=-0.5$ (low porosity) and wall dilation rate $\alpha=0.5,-0.5$ (expansion and contraction, resp.) over a range of $R_{e}$. In case of $R_{e}>0$, Figures 11 and 12 show that increasing injection leads to a higher self-axial velocity near the center and a lower one near the wall. For $R_{e}<0$, Figures 11 and 12 show that increasing suction ratio leads to a lower self-axial velocity at the center and a higher one near the wall. A comparison between Figures 11 and 12 shows that the self-axial velocity near the center in case of 


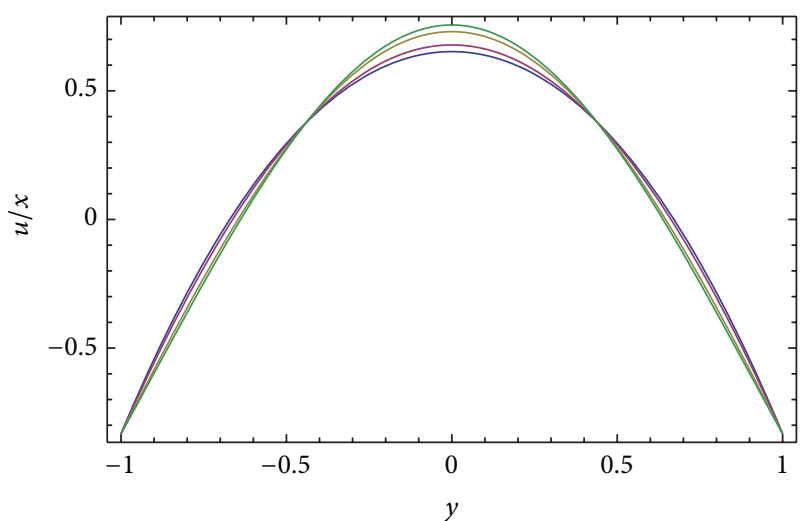

FIGURE 9: Self-axial velocity profiles over a range of $R_{e}$, where blue $=$ 1 , pink $=0.5$, yellow $=-0.5$, and green $=-1$ at $\alpha=0.5$ and $R=0.5$.

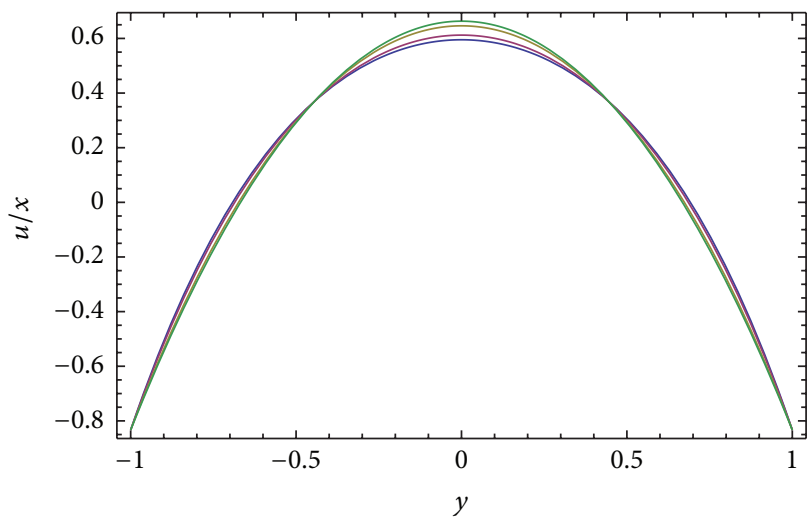

FIGURE 10: Self-axial velocity profiles over a range of $R_{e}$, where blue $=1$, pink $=0.5$, yellow $=-0.5$, and green $=-1$ at $\alpha=-0.5$ and $R=0.5$.

TABLE 1: Comparison between analytical and numerical solutions for self-axial velocity $u / x$ at $y=0.1$ for $R_{e}=1, \alpha=0.5$.

\begin{tabular}{lccc}
\hline & $\begin{array}{c}\text { Analytical } \\
\text { method }\end{array}$ & $\begin{array}{c}\text { Numerical } \\
\text { method }\end{array}$ & $\begin{array}{c}\text { Percentage } \\
\text { error (\%) }\end{array}$ \\
\hline$R=-1$ & 1.549755 & 1.549040 & 0.046138 \\
$R=-0.5$ & 1.575774 & 1.576305 & 0.033691 \\
$R=0.5$ & 1.471699 & 1.480956 & 0.625085 \\
$R=1$ & 1.497718 & 1.501653 & 0.262055 \\
\hline
\end{tabular}

TABLE 2: Comparison between analytical and numerical solutions for self-axial velocity $u / x$ at $y=0.1$. for $R_{e}=1, \alpha=-0.5$.

\begin{tabular}{lccc}
\hline & $\begin{array}{c}\text { Analytical } \\
\text { method }\end{array}$ & $\begin{array}{c}\text { Numerical } \\
\text { method }\end{array}$ & $\begin{array}{c}\text { Percentage } \\
\text { error (\%) }\end{array}$ \\
\hline$R=-1$ & 1.480690 & 1.480513 & 0.012005 \\
$R=-0.5$ & 1.501697 & 1.503207 & 0.100457 \\
$R=0.5$ & 1.417671 & 1.423694 & 0.423027 \\
$R=1$ & 1.438678 & 1.440991 & 0.160530 \\
\hline
\end{tabular}

injection with expanding wall and low porosity is higher than injection with contracting wall and low porosity.

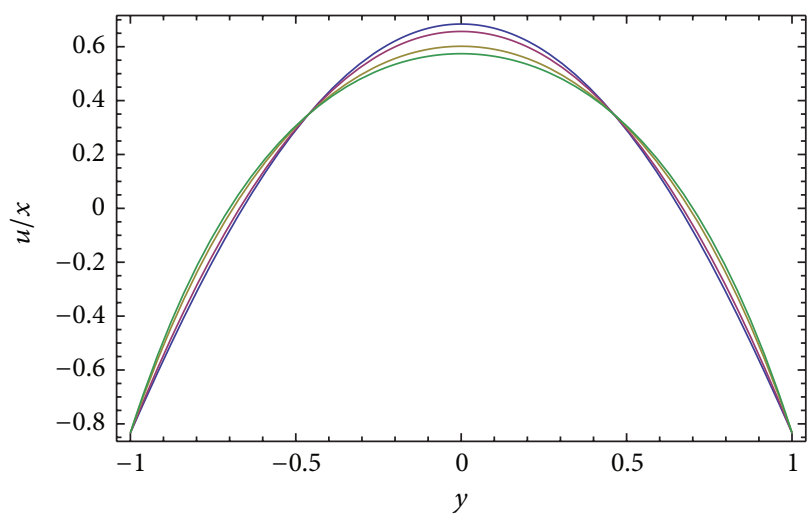

FIGURE 11: Self-axial velocity profiles over a range of $R_{e}$, where blue $=$ 1 , pink $=0.5$, yellow $=-0.5$, and green $=-1$ at $\alpha=-0.5$ and $R=-0.5$.

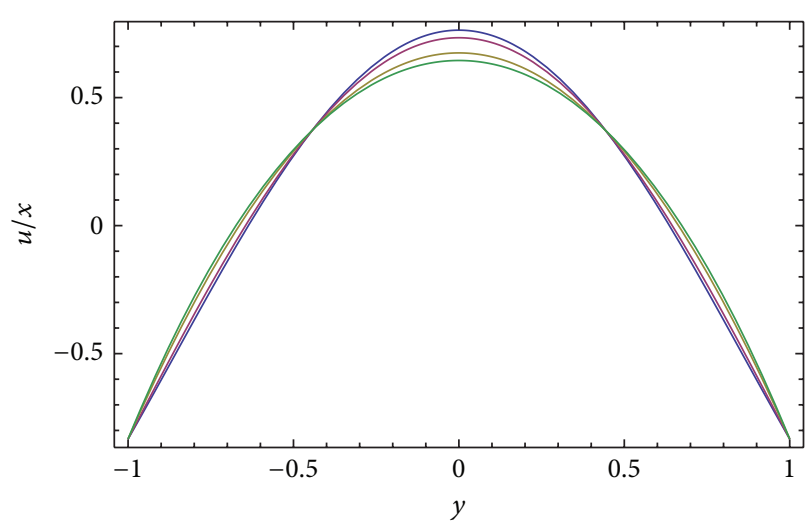

FIGURE 12: Self-axial velocity profiles over a range of $R_{e}$, where blue $=1$, pink $=0.5$, yellow $=-0.5$, and green $=-1$ at $\alpha=0.5$ and $R=-0.5$.

TABLE 3: Comparison between analytical and numerical solutions for self-axial velocity $u / x$ at $y=0.1$ for $R_{e}=-1, \alpha=0.5$.

\begin{tabular}{lccc}
\hline & $\begin{array}{c}\text { Analytical } \\
\text { method }\end{array}$ & $\begin{array}{c}\text { Numerical } \\
\text { method }\end{array}$ & $\begin{array}{c}\text { Percentage } \\
\text { error (\%) }\end{array}$ \\
\hline$R=-1$ & 1.490782 & 1.490460 & 0.012660 \\
$R=-0.5$ & 1.464764 & 1.466093 & 0.090696 \\
$R=0.5$ & 1.568838 & 1.583640 & 0.934648 \\
$R=1$ & 1.542820 & 1.548592 & 0.372749 \\
\hline
\end{tabular}

TABLE 4: Comparison between analytical and numerical solutions for self-axial velocity $u / x$ at $y=0.1$ for $R_{e}=-1, \alpha=-0.5$.

\begin{tabular}{lccc}
\hline & $\begin{array}{c}\text { Analytical } \\
\text { method }\end{array}$ & $\begin{array}{c}\text { Numerical } \\
\text { method }\end{array}$ & $\begin{array}{c}\text { Percentage } \\
\text { error (\%) }\end{array}$ \\
\hline$R=-1$ & 1.418772 & 1.419039 & 0.018778 \\
$R=-0.5$ & 1.397766 & 1.399917 & 0.153681 \\
$R=0.5$ & 1.481791 & 1.491195 & 0.630644 \\
$R=1$ & 1.460785 & 1.464232 & 0.235434 \\
\hline
\end{tabular}

The self-axial velocity $u / x$ for porosity parameter $R=$ $-0.5,0.5$ (low and high porosity, resp.,) and wall dilation rate 
TABLE 5: Comparison between analytical and numerical solutions for self-axial velocity $u / x$ at $y=0.1$ for $R=1, R_{e}=1$.

\begin{tabular}{lccc}
\hline & $\begin{array}{c}\text { Analytical } \\
\text { method }\end{array}$ & $\begin{array}{c}\text { Numerical } \\
\text { method }\end{array}$ & $\begin{array}{c}\text { Percentage } \\
\text { error (\%) }\end{array}$ \\
\hline$\alpha=-1$ & 1.409157 & 1.412797 & 0.257641 \\
$\alpha=-0.5$ & 1.438678 & 1.440991 & 0.160530 \\
$\alpha=0.5$ & 1.497718 & 1.501653 & 0.262055 \\
$\alpha=1$ & 1.527238 & 1.534003 & 0.440984 \\
\hline
\end{tabular}

TABLE 6: Comparison between analytical and numerical solutions for self-axial velocity $u / x$ at $y=0.1$ for $R=1, R_{e}=-1$.

\begin{tabular}{lccc}
\hline & $\begin{array}{c}\text { Analytical } \\
\text { method }\end{array}$ & $\begin{array}{c}\text { Numerical } \\
\text { method }\end{array}$ & $\begin{array}{c}\text { Percentage } \\
\text { error (\%) }\end{array}$ \\
\hline$\alpha=-1$ & 1.419768 & 1.426770 & 0.490778 \\
$\alpha=-0.5$ & 1.460785 & 1.464232 & 0.235434 \\
$\alpha=0.5$ & 1.542820 & 1.548592 & 0.372749 \\
$\alpha=1$ & 1.583837 & 1.595620 & 0.738487 \\
\hline
\end{tabular}

TABLE 7: Comparison between analytical and numerical solutions for self-axial velocity $u / x$ at $y=0.1$ for $R=-1, R_{e}=-1$.

\begin{tabular}{lccc}
\hline & $\begin{array}{c}\text { Analytical } \\
\text { method }\end{array}$ & $\begin{array}{c}\text { Numerical } \\
\text { method }\end{array}$ & $\begin{array}{c}\text { Percentage } \\
\text { error (\%) }\end{array}$ \\
\hline$\alpha=-1$ & 1.382767 & 1.387131 & 0.314608 \\
$\alpha=-0.5$ & 1.418772 & 1.419039 & 0.018778 \\
$\alpha=0.5$ & 1.490782 & 1.490460 & 0.021660 \\
$\alpha=1$ & 1.526788 & 1.530071 & 0.214601 \\
\hline
\end{tabular}

TABLE 8: Comparison between analytical and numerical solutions for self-axial velocity $u / x$ at $y=0.1$ for $R=-1, R_{e}=1$.

\begin{tabular}{lccc}
\hline & $\begin{array}{c}\text { Analytical } \\
\text { method }\end{array}$ & $\begin{array}{c}\text { Numerical } \\
\text { method }\end{array}$ & $\begin{array}{c}\text { Percentage } \\
\text { error (\%) }\end{array}$ \\
\hline$\alpha=-1$ & 1.446158 & 1.448653 & 0.172244 \\
$\alpha=-0.5$ & 1.480690 & 1.480513 & 0.012005 \\
$\alpha=0.5$ & 1.549755 & 1.549040 & 0.046138 \\
$\alpha=1$ & 1.584287 & 1.585538 & 0.078889 \\
\hline
\end{tabular}

$\alpha=-0.5$ (contraction) over a range of $R_{e}$ has been explained in Figures 10 and 11. When $R_{e}>0$, Figure 10 shows that increasing injection leads to a lower self-axial velocity near the center and a higher one near the wall. Figure 11 shows that increasing injection leads to a higher self-axial velocity near the center and a lower one near the wall. In case of $R_{e}<0$, Figure 10 shows that increasing suction ratio leads to a higher self-axial velocity at the center and a lower one near the wall. Increasing suction ratio leads to a lower self-axial velocity at the center and a higher one near the wall (Figure 11). A comparison shows that the self-axial velocity near the center in case of injection with contracting wall and low porosity is higher than injection with contracting wall and high porosity (Figures 10 and 11).

Figures 9 and 12 indicate the behaviour of self-axial velocity $u / x$ for porosity parameter $R=-0.5,0.5$ (low and high porosity, resp.) and wall dilation rate $\alpha=0.5$
TABLE 9: Comparison between analytical and numerical solutions for self-axial velocity $u / x$ at $y=0.1$ for $R=1, \alpha=-0.5$.

\begin{tabular}{lccc}
\hline & $\begin{array}{c}\text { Analytical } \\
\text { method }\end{array}$ & $\begin{array}{c}\text { Numerical } \\
\text { method }\end{array}$ & $\begin{array}{c}\text { Percentage } \\
\text { error (\%) }\end{array}$ \\
\hline$R_{e}=-1$ & 1.460785 & 1.464232 & 0.235434 \\
$R_{e}=-0.5$ & 1.455288 & 1.456930 & 0.114768 \\
$R_{e}=0.5$ & 1.444204 & 1.445507 & 0.090102 \\
$R_{e}=1$ & 1.438678 & 1.440991 & 0.160530 \\
\hline
\end{tabular}

TABLE 10: Comparison between analytical and numerical solutions for self-axial velocity $u / x$ at $y=0.1$ for $R=1, \alpha=0.5$.

\begin{tabular}{lccc}
\hline & $\begin{array}{c}\text { Analytical } \\
\text { method }\end{array}$ & $\begin{array}{c}\text { Numerical } \\
\text { method }\end{array}$ & $\begin{array}{c}\text { Percentage } \\
\text { error (\%) }\end{array}$ \\
\hline$R_{e}=-1$ & 1.542820 & 10548592 & 0.372749 \\
$R_{e}=-0.5$ & 1.531544 & 1.533740 & 0.143138 \\
$R_{e}=0.5$ & 1.508993 & 1.510721 & 0.114334 \\
$R_{e}=1$ & 1.497718 & 1.501653 & 0.262055 \\
\hline
\end{tabular}

TABLE 11: Comparison between analytical and numerical solutions for self-axial velocity $u / x$ at $y=0.1$ for $R=-1, \alpha=-0.5$.

\begin{tabular}{lccc}
\hline & $\begin{array}{c}\text { Analytical } \\
\text { method }\end{array}$ & $\begin{array}{c}\text { Numerical } \\
\text { method }\end{array}$ & $\begin{array}{c}\text { Percentage } \\
\text { error (\%) }\end{array}$ \\
\hline$R_{e}=-1$ & 1.418772 & 1.419039 & 0.018778 \\
$R_{e}=-0.5$ & 1.434252 & 1.435114 & 0.060097 \\
$R_{e}=0.5$ & 1.465211 & 1.465912 & 0.048020 \\
$R_{e}=1$ & 1.480690 & 1.480513 & 0.012005 \\
\hline
\end{tabular}

TABLE 12: Comparison between analytical and numerical solutions for self-axial velocity $u / x$ at $y=0.1$ for $R=-1, \alpha=0.5$.

\begin{tabular}{lccc}
\hline & $\begin{array}{c}\text { Analytical } \\
\text { method }\end{array}$ & $\begin{array}{c}\text { Numerical } \\
\text { method }\end{array}$ & $\begin{array}{c}\text { Percentage } \\
\text { error (\%) }\end{array}$ \\
\hline$R_{e}=-1$ & 1.490782 & 1.490460 & 0.021660 \\
$R_{e}=-0.5$ & 1.505526 & 1.506243 & 0.047638 \\
$R_{e}=0.5$ & 1.535012 & 1.535561 & 0.035738 \\
$R_{e}=1$ & 1.523217 & 1.524206 & 0.046138 \\
\hline
\end{tabular}

(expansion) over a range of $R_{e}$. In case of $R_{e}>0$, Figure 9 shows that increasing injection leads to a lower self-axial velocity near the center and a higher one near the wall. Figure 12 shows that increasing injection leads to a higher self-axial velocity near the center and a lower one near the wall. In case of $R_{e}<0$, Figure 9 depicts that increasing suction ratio leads to a higher self-axial velocity at the center and a lower one near the wall. Figure 12 shows that increasing suction ratio leads to a lower self-axial velocity at the center and a higher one near the wall. By comparing Figures 9 and 12 , the self-axial velocity near the center in case of injection with expansion wall and low porosity is higher than injection with expansion wall and large porosity.

Tables $9,10,11$, and 12 show the percentage error decrease for a small $R_{e}$.

The plots in Figure 13 elucidate that the temperature distribution is constant throughout and it is independent of 


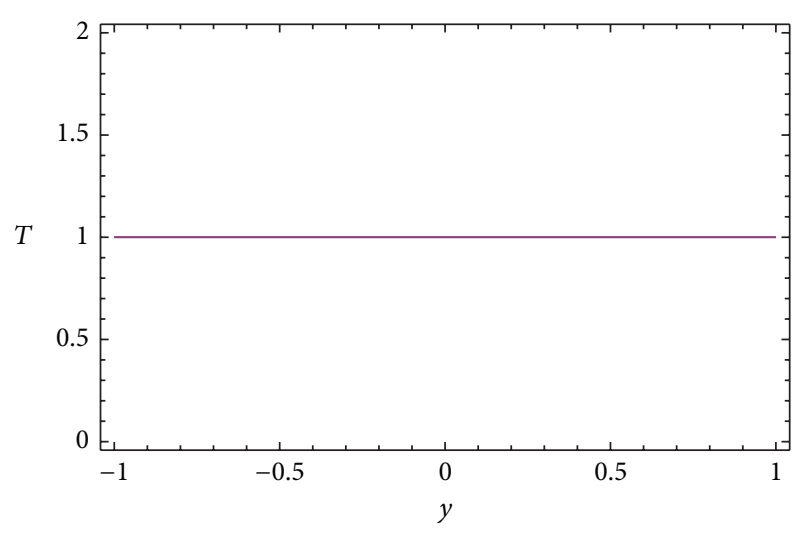

FIGURE 13: Temperature distribution profile.

physical parameter. Numerical solution for temperature is similar to our analytical solution, and therefore, temperature distribution has no error.

\section{Conclusions}

In this paper, we have generalized the flow analysis of [4] with the influence of porous medium and heat transfer. Analytical solution for the arising nonlinear problem is obtained by using Lie symmetry technique in conjunction with a secondorder double perturbation method. We have studied the effects of porous medium $(R)$, permeation Reynolds $R_{e}$, and wall dilation rate $\alpha$ on the self-axial velocity and temperature distribution within the fluid. We compared the analytical solution with the numerical solution for self-axial velocity for the different values of $R, R_{e}$, and $\alpha$.

It was found that the temperature distribution has no error since analytical solution is similar to numerical solution and both are equal to one. We also found that as $R$ increases, the percentage error decreases and that temperature distribution is constant throughout. Here, we have noticed that the obtained analytical results match quite well with the numerical results for a good range of these parameters. We also noticed that in all cases, the self-axial velocity has similar trend as in [4], that is, the self-axial velocity approaches a cosine profile. Finally, we observed that when $R$ approaches infinity, our problem reduces to the problem in [4] and our results (analytical and numerical) also reduce to the results in $[4]$.

\section{References}

[1] A. S. Berman, "Laminar flow in channels with porous walls," Journal of Applied Physics, vol. 24, pp. 1232-1235, 1953.

[2] E. C. Dauenhauer and J. Majdalani, "Exact self similarity solution of the Navier-Stokes equations for a deformable channel with wall suction or injection," The American Institute of Aeronautics and Astronautics, vol. 3588, pp. 1-11, 2001.

[3] J. Majdalani, C. Zhou, and C. A. Dawson, "Two-dimensional viscous flow between slowly expanding or contracting walls with weak permeability," Journal of Biomechanics, vol. 35, no. 10, pp. 1399-1403, 2002.
[4] Y. Z. Boutros, M. B. Abd-el-Malek, N. A. Badran, and H. S. Hassan, "Lie-group method solution for two-dimensional viscous flow between slowly expanding or contracting walls with weak permeability," Applied Mathematical Modelling, vol. 31, no. 6, pp. 1092-1108, 2007.

[5] M. Mahmood, M. A. Hossain, S. Asghar, and T. Hayat, "Application of homotopy perturbation method to deformable channel with wall suction and injection in a porous medium," International Journal of Nonlinear Sciences and Numerical Simulation, vol. 9, no. 2, pp. 195-206, 2008.

[6] S. Asghar, M. Mushtaq, and A. H. Kara, "Exact solutions using symmetry methods and conservation laws for the viscous flow through expanding-contracting channels," Applied Mathematical Modelling, vol. 32, no. 12, pp. 2936-2940, 2008.

[7] D. Z. Noor, P. R. Kanna, and M.-J. Chern, "Flow and heat transfer in a driven square cavity with double-sided oscillating lids in anti-phase," International Journal of Heat and Mass Transfer, vol. 52, no. 13-14, pp. 3009-3023, 2009. 


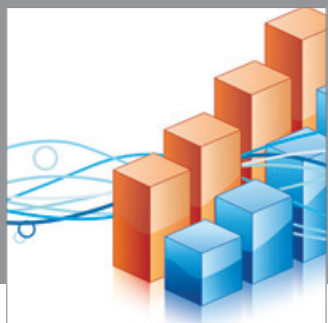

Advances in

Operations Research

mansans

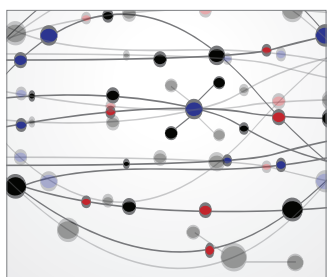

The Scientific World Journal
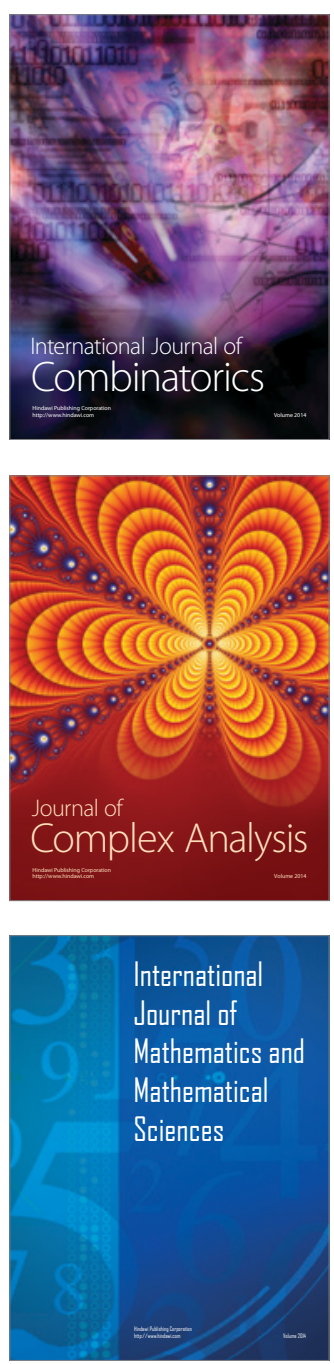
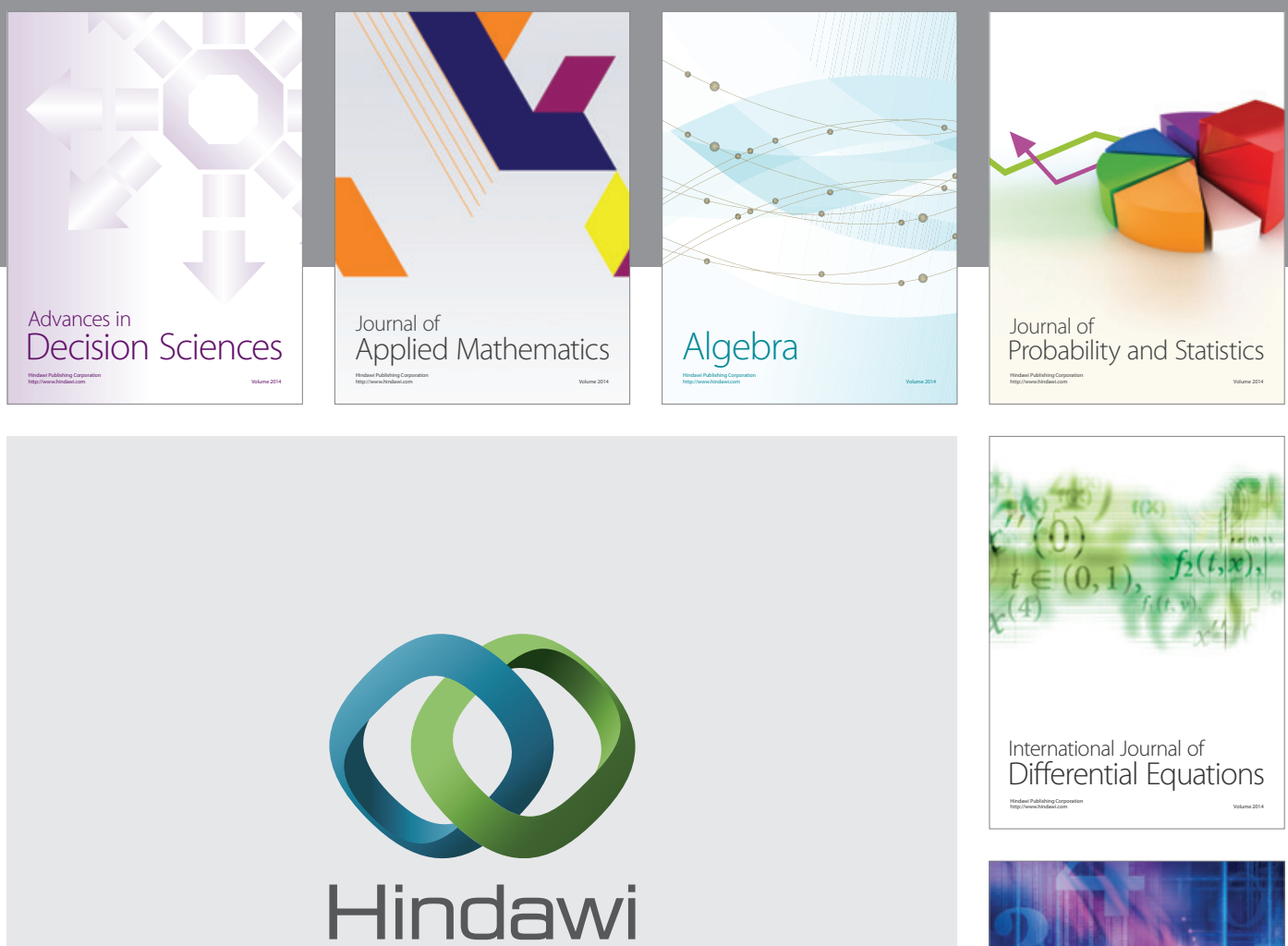

Submit your manuscripts at http://www.hindawi.com
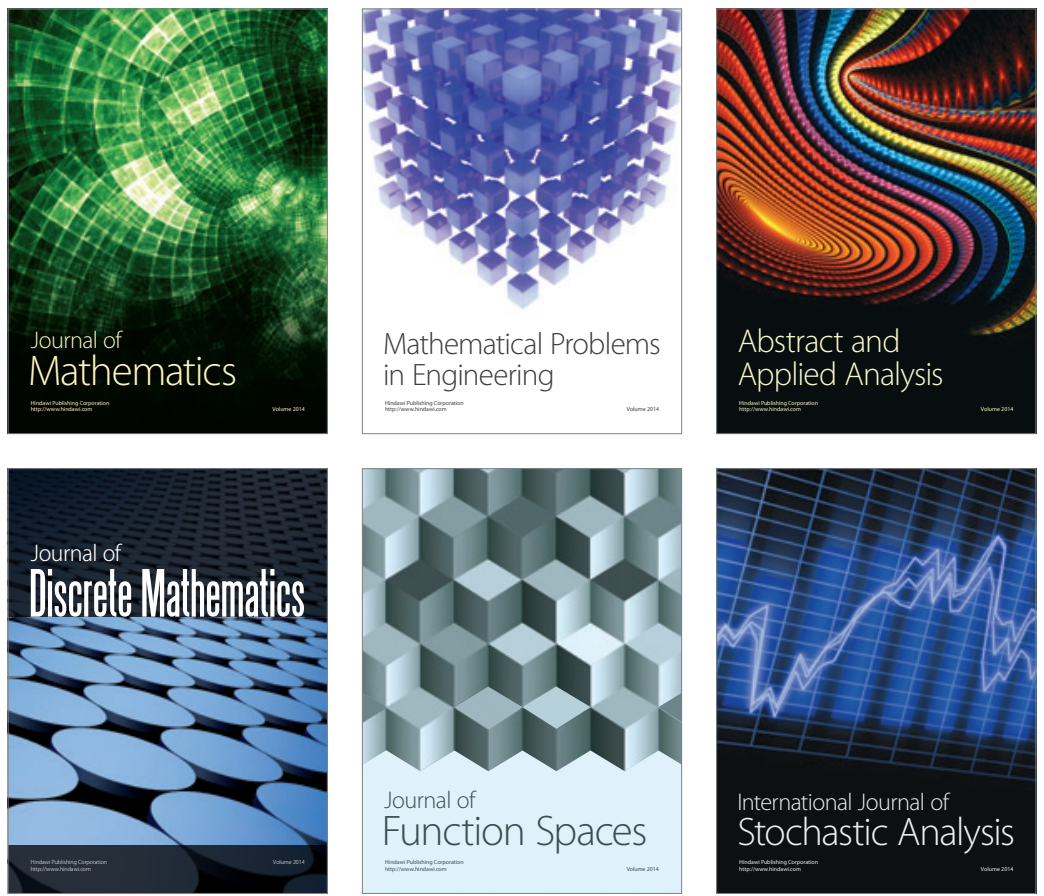

Journal of

Function Spaces

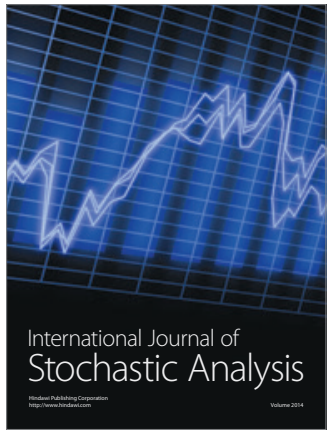

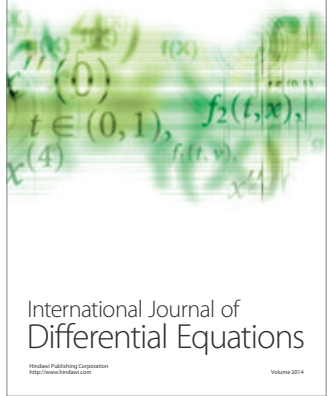
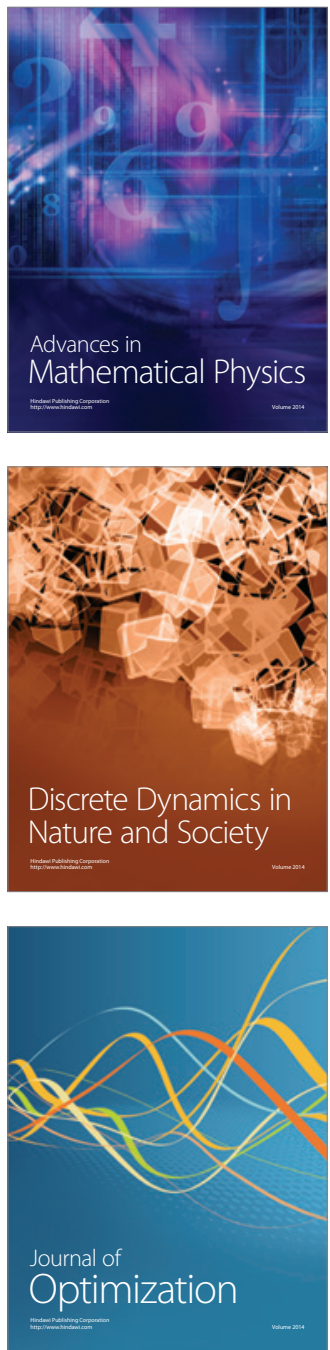